\section{Age-dependent Role of Cilostazol on Cold Restraint Stress- induced Gastric Ulceration in Female Rats}

\author{
Abeer Mohamed Rashad ${ }^{1^{*}}$, Gehan Hussein Heeba ${ }^{2}$, Somya Hassan Hamad ${ }^{2}$
}

${ }^{1}$ Department of Pharmacology, Faculty of Medicine, Assiut University, Egypt

${ }^{2}$ Department of Pharmacology and Toxicology, Faculty of Pharmacy, Minia University, Egypt

\section{Correspondence:}

Abeer Mohamed Rashad

Address: Department of Pharmacology and Toxicology, Faculty of Pharmacy, Minia University, Egypt

Email: abeermrh@yahoo.com

\begin{abstract}
Introduction: Cilostazol (CIL), a phosphodiesterase III inhibitor, is a potent antiplatelet drug which possesses vasodilator and anti-inflammatory activity that making it an effective drug in gastric ulcer protection.

Objectives: This study investigated the influence of age on the effects of CIL against cold restraint stress (CRS) - induced gastric ulcer in rats.

Methods: CIL (10 mg/kg/day) was administered orally for 2 weeks to adult and aged female rats before CRS-induced gastric ulcer.

Results: This syudy showed that CIL exhibited gastroprotective effects in both adult and aged rats as evidenced by significant decrease in ulcer index, gastric mucosal malondialdehyde level, myeloperoxidase activity and tumor necrosis factor- $\alpha$ protein expression with concomitant increase in gastric mucosal reduced glutathione level and glutathione peroxidase activity, nitric oxide, prostaglandin $\mathrm{E}_{2}$ level, cyclooxygenase- 1 and 2 protein expression and gastric juice mucin concentration compared to adult and aged CRS rats, respectively. Additionally, the histopathological examination results came to confirm the protection afforded by CIL in CRS - induced gastric ulcer.
\end{abstract}

Conclusion: The possible protective effect of CIL against CRS-induced gastric ulceration in both adult and aged rat's may be explained via its antioxidant and antiinflammatory properties and by enhancement of gastric mucus secretion. It could be recommended that CIL is considered as a more proper antiplatelet drug for adult and elderly patients who are at risk of gastric ulceration.

Keywords: cilostazol, gastric ulcer, nitric oxide, prostaglandin $E_{2}$, cyclooxygenase

\section{INTRODUCTION}

Stress-induced gastric ulceration is a severe damage occurring as a complication of exposure to excessive stress, for example, burns, organ failure, major surgery and sepsis [1]. Cold restraint stress (CRS) is the commonly applicable method used for induction of acute gastric ulcer in experimental animals. Maintenance of blood flow is important for the protection of gastric mucosa from endogenous and exogenous damaging factors and a sudden reduction in the gastric mucosal blood flow and increased free radical production play vital roles in gartric ulcer formation [2].

Many previous reports indicated that gastric mucosa in aged individuals has impaired mucosal defense by decreasing mucus, bicarbonate and prostaglandins (PGs) production, and reducing of nitric oxide synthase (NOS) activity and consequently impairing gastric mucosal blood flow [3-5].

Antiplatelet therapy is useful in ameliorating the incidence of myocardial infarction, cerebrovascular accidents and death from vascular causes in individuals with thromboembolic diseases [6]. Aspirin in low-dose (75-325 mg/day) is most commonly used for the primary and secondary prevention of vascular thrombosis. As a consequence of their action on cyclooxygenase (COX) enzymes, aspirin and other non-steroidal anti-inflammatory drugs (NSAIDs) are associated with upper 
gastrointestinal (GI) adverse effects, including ulceration and bleeding [7]. Moreover, a clinical trial has reported the superiority of combined antiplatelet treatment over single aspirin administration in preventing thrombotic consequences [8].

Cilostazol (CIL), antiplatelet drug, acts by inhibition of phosphodiesterase (PDE) III which possesses vasodilator, anti-oxidant and anti-inflammatory effects $[9,10,11]$. It was first approved for the treatment of intermittent claudication (IC) [9]. Accordingly, this study investigated the role of cilostazol on CRS-induced gastric ulceration in adult and aged rats. In addition, we aimed to evaluate the possible mechanisms involved in the effect of this drug.

\section{MATERIALS AND METHODS}

\section{Drugs and Chemicals}

Cilostazol (CIL) was obtained from Otsuka pharmaceutical company, Egypt. Enzyme-linked immunosorbent assay (ELISA) kit for assessment of gastric mucosal prostaglandin E2 (PGE2) was provided by Elabscience Biotechnology Co., Ltd (Hubei, China). Antibodies against cyclooxygenase-1 (COX-1), cyclooxygenase-2 (COX-2) and tumor necrosis factor-alpha (TNF- $\alpha$ ) were purchased from Thermo Fisher Scientific (Rockford, USA). $\beta$-actin antibodies were purchased from Novus Biologicals (Littleton, CO, USA). Other chemicals were of analytical grade and were obtained from commercial sources.

\section{Animals}

Adult female Wister rats of 3 months age (weighing 170$200 \mathrm{~g}$ ) and aged female Wister rats of 24 months age (weighing 250-300 g) were obtained from animal house, Assiut University, Assiut, Egypt. Female rats were selected in this study to ensure their ageing by stoppage their estrus cycle. Animals were used after two weeks of accommodation to the animal house conditions ( $12 \mathrm{~h}$ lighting cycle and $25 \pm$ $2^{\circ} \mathrm{C}$ temperature) and had free access to standard rodent chow and water. The animals care was done in accordance with the guidelines of the Canadian Council on Animal Care (Vol. 1, 2nd ed., 1993, and Vol. 2, 1984, available from the Canadian Council on Animal Care, Constitution Square, Tower 2, Suite 315, 350 Albert Street, Ottawa, ON K1R 1B1, Canada, or on their Web site at www.ccac.ca).

\section{Experimental Design and Induction of CRS Ulcer}

Adult and aged rats were divided into 4 groups randomly (8 rats per each). The first group is the normal control (adult control or aged control), in which rats were left freely moving in their cages. The second group is served as control CIL (adult control CIL or aged control CIL), in which rats were left freely moving in their cages and treated with CIL once daily for 2 weeks. The third group is an adult or aged CRS group, in which rats were exposed to CRS ulcer. The fourth group is an adult CRS + CIL or aged CRS + CIL, in which rats pretreated with CIL once daily for 2 weeks before CRS ulcer.

CIL was suspended in $2 \%$ Arabic gum solution and orally (P.O.) given at a dose of $10 \mathrm{mg} / \mathrm{kg}$ once daily for 2 weeks in a volume of $5 \mathrm{ml} / \mathrm{kg}$ [12].

Twenty four hours before the experiment, rats were deprived of food in a mesh-bottomed cages with free access to water until the last two hours before the experiment. CRS ulcer was induced in rats by fixing the four limbs to a wooden board and they were placed in a refrigerator at $4^{\circ} \mathrm{C}$ for $3 \mathrm{~h}$ [2]. All experiments were performed during the same time of the day to avoid diurnal variations of the reputed gastric functions regulators.

\section{Collection and Analysis of Gastric Juice and Mucosa}

At the end of the experiment animals were sacrificed. The rats were dissected and after opening the stomach and the gastric juice was collected. The collected gastric juice was centrifuged for 15 minutes at $3000 \mathrm{rpm}$ and the supernatant was used for determination of gastric mucin concentration [13].

The stomachs were washed with cold saline and the gross mucosal lesions were scored that expressed in terms of ulcer index (U.I.) as previously described [14]. The gastric mucosa was scraped and immediately stored at $-80 \mathrm{oC}$ for assessment of mucosal content of malondialdehyde (MDA) [15], myeloperoxidase (MPO) enzyme activity [16], reduced glutathione (GSH) [17], glutathione peroxidase activity (GPx) [18], nitric oxide (NO) concentration [19] and PGE2 concentration by using ELISA PGE2 immunoassay kit.

\section{Western Blot Assay}

Gastric mucosal tissue samples were homogenized in lysis buffer $(20$ mMTris- $\mathrm{HCl} \quad \mathrm{pH} \quad 7.5, \quad 50 \mathrm{mM} \quad 2-$ mercaptoethanol, $5 \mathrm{mM}$ EGTA, $2 \mathrm{mM}$ EDTA, 1\% NP40, $0.1 \%$ SDS, $0.5 \%$ deoxycholic acid, $10 \mathrm{mMNaF}, 1 \mathrm{mM}$ PMSF, $25 \mathrm{mg} / \mathrm{mL}$ leupeptin, $2 \mathrm{mg} / \mathrm{mL}$ aprotinin) and protein concentrations were determined using Bradford dyebinding method [20]. For immunoblotting, aliquots of lysate were mixed with a loading buffer containing 2mercaptoethanol (at $100{ }^{\circ} \mathrm{C}$ for $10 \mathrm{~min}$ ) then loading on $10 \%$ SDS-PAGE. Following SDS-PAGE separation, proteins were transferred to PVDF membrane. Membranes were blocked in TBST containing 5\% non-fat milk for $1 \mathrm{~h}$ at room temperature. Membrane strips were incubated with primary antibodies (diluted 1:1000) overnight at $4{ }^{\circ} \mathrm{C}$. Following excessive washing, membrane strips were incubated with anti-rabbit IgG (1:5000; Cell Signaling Technology Inc., USA) conjugated with horseradish peroxidase for $1 \mathrm{~h}$. Protein bands were detected by a chemiluminescence method and densitometry measurements were made using Image J software (freeware; www.rsbweb.nih.gov/ij).

\section{Histolopathological Examination}

Gastric specimens were taken from all groups and were fixed in formalin $10 \%$. Paraffin sections were prepared and 


\section{Adult groups}

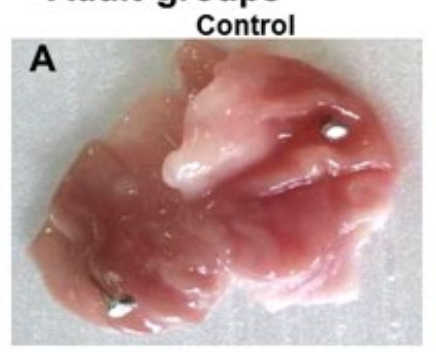

\section{Aged groups}

Control

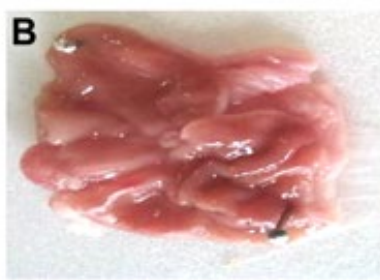

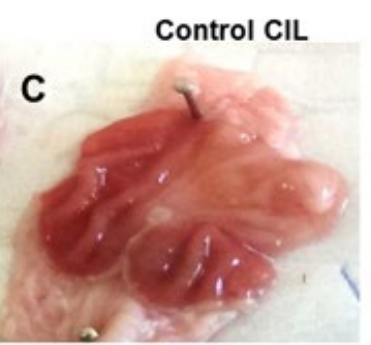
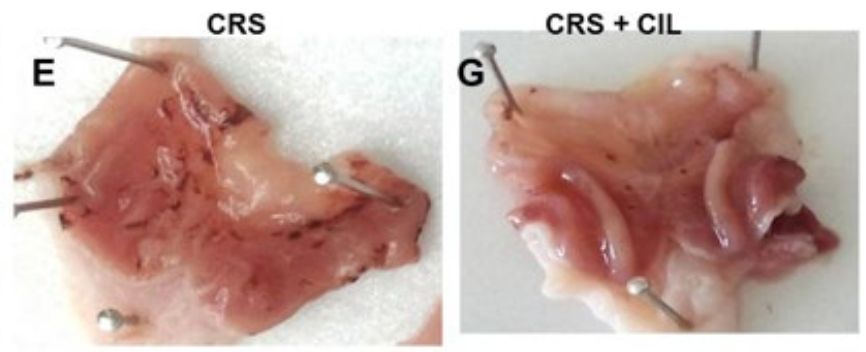

Control CIL
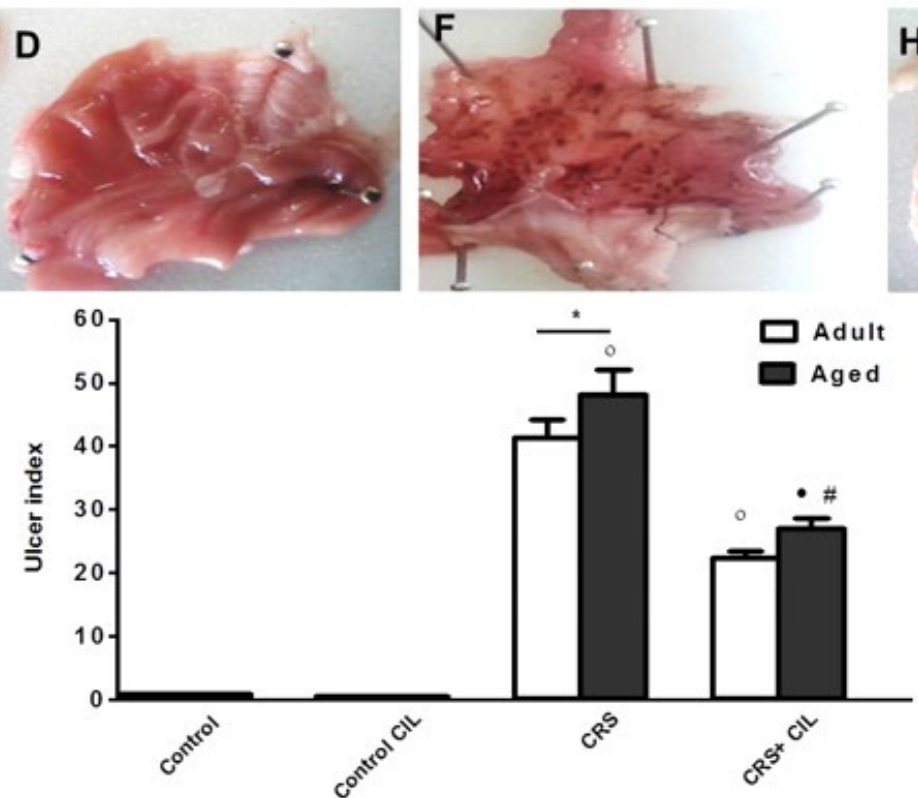
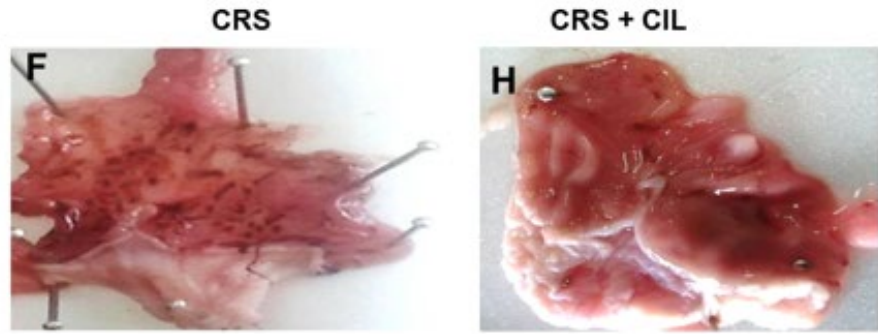

\section{年}

\section{Figure 1. Effect of CIL on CRS-induced gastric lesions in adult and aged rats}

Figure shows original captured images displaying ulcers or injuries in the mucous membrane area in adult and aged control rats ( $A$ and $B$, respectively), adult and aged control $\mathrm{CIL}$ rats ( $C$ and $\mathrm{D}$, respectively), adult and aged CRS-induced gastric ulcer rats ( $\mathrm{E}$ and $\mathrm{F}$, respectively), adult and aged $\mathrm{CRS}+\mathrm{CIL}$ rats (G and $\mathrm{H}$, respectively) and representative bar chart of ulcer score in all groups (I).

Data are mean \pm SEM of 6 rats.

"Significantly different from normal control rats at $p<0.05$.

oSignificantly different from adult CRS rats at $p<0.05$.

- Significantly different from aged CRS rats at $p<0.05$

\#Significantly different from adult CRS + CIL rats at $p<0.05$.

CRS: Cold restraint stress; CIL: Cilostazol.

stained with Haematoxylin and Eosin. The histopathological examination was undertaken by light microscopy.

\section{Statistical Analysis}

Results were presented as means \pm standard error of the mean (SEM) and were analyzed using one-way analysis of variance (ANOVA) followed by the post analysis test for statistically significant difference. $\mathrm{P}$ values $<0.05$ were considered significant. GraphPad Prism ${ }^{\circledast}$ was used for statistical calculations (Version 7.00, GraphPad Software, San Diego California USA, www.graphpad.com).

RESULTS
Effect of CIL on CRS-induced Gastric Lesions in
Adult and Aged Rats
Results revealed that CRS produced ulcerative lesions in
the glandular portion of the stomach and a significant ( $p$
$<0.05)$ increase was observed in the U.I in both adult (41.40
$\pm 2.86)$ and aged $(48.15 \pm 3.82)$ CRS rats compared to control

adult $(0.810 \pm 0.001)$ and control aged $(0.920 \pm 0.001)$ rats, respectively with more significant increase in aged CRS rats compared to adult CRS group (Figure 1). In contrast, pretreatment with CIL showed a significant $(\mathrm{p}<0.05)$ decrement in U.I. in both adult $(22.38 \pm 1.13)$ and aged $(27.03 \pm 1.61)$ CRS rats compared to their respective CRS groups, respectively (Figure 1).

\section{Effect of CIL on Gastric Mucosal Oxidative Stress}

\section{Parameters in Adult and Aged Rats}

CRS significantly $(\mathrm{p}<0.05)$ increased gastric mucosal MDA concentration and MPO activity while, significantly ( $\mathrm{p}$ $<0.05)$ decreased glutathione peroxidase activity and reduced glutathione concentration in both groups compared to control adult and aged rats, respectively (Figure 2). Also, the results denoted that there is more significant effect in aged CRS rats compared to adult CRS group. Furthermore, the findings in Figure 2 indicated that CIL pretreatment 

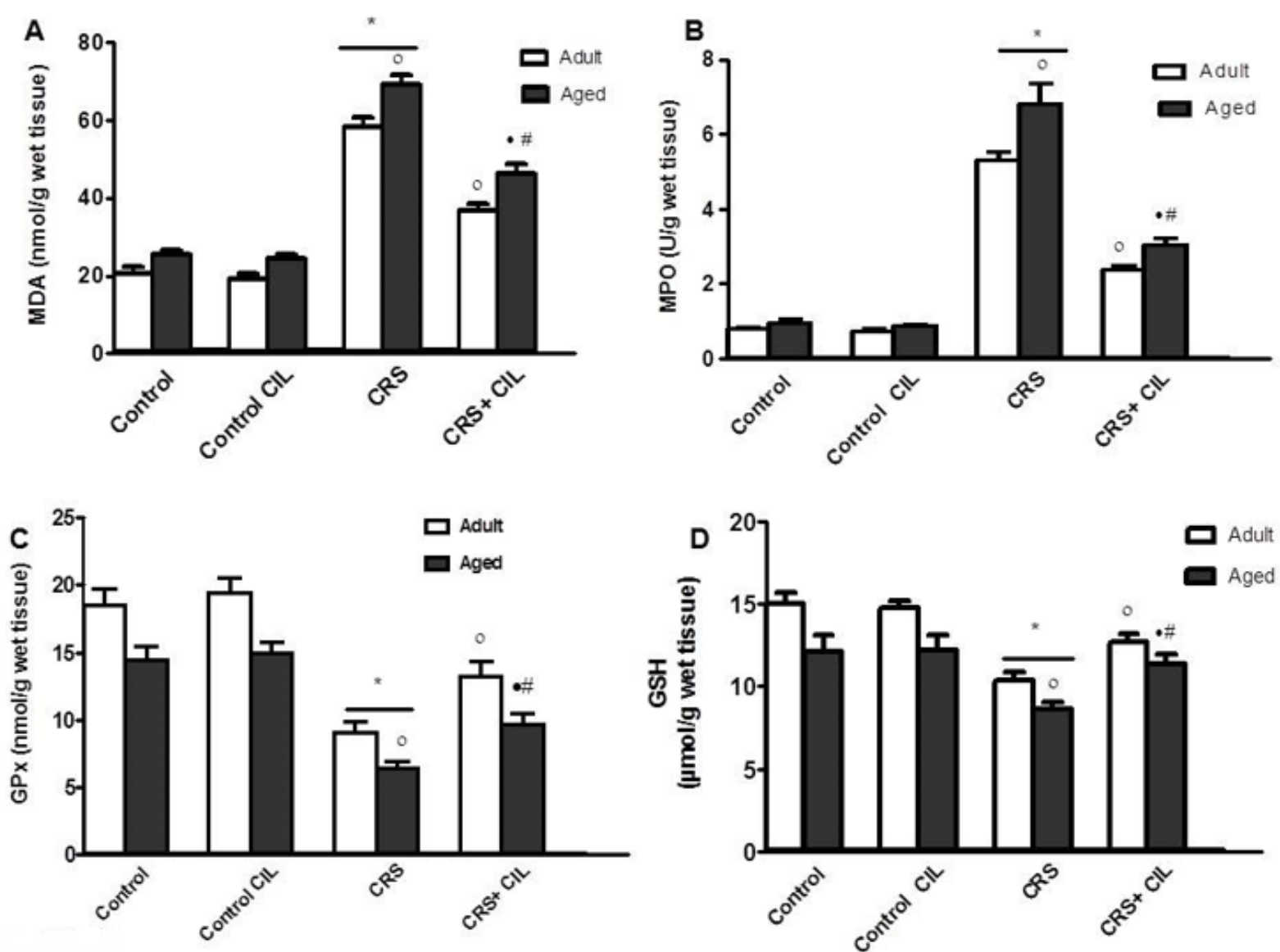

Figure 2. Effect of CIL on gastric mucosal MDA (A), MPO (B), GPx (C) and GSH (D) in CRS-induced gastric ulcer in adult and aged rats Data are mean \pm SEM of 6 rats.

"Significantly different from normal control rats at $p<0.05$.

o Significantly different from adult CRS rats at $p<0.05$.

- Significantly different from aged CRS rats at $p<0.05$.

"Significantly different from adult CRS $+\mathrm{CIL}$ rats at $\mathrm{p}<0.05$

CRS: Cold restraint stress; CIL: Cilostazol.

alleviated these parameters with less significant $(\mathrm{p}<0.05)$ improvement in aged CRS + CIL rats compared to adult CRS + CIL group.

\section{Effect of CIL on Gastric Mucosal COX-1 \& 2 Protein} Expressions in Adult and Aged Rats

The result of this study illustrated a significant $(\mathrm{p}<0.05)$ elevation in the gastric mucosal COX-1 \& 2 protein expression following CRS induction in both adult and aged groups compared to their respective control groups. This increase was more significant in aged CRS - induced group compared to adult CRS group (Figure 3). However, pretreatment with CIL produced a significant $(\mathrm{p}<0.05)$ increase in COX-1 \& 2 protein expression in adult and aged CRS + CIL groups compared to their respective CRS induced groups. This increase was more significant $(\mathrm{p}<0.05)$ in aged CRS + CIL group compared to adult CRS + CIL group (Figure 3 ).

\section{Effect of CIL on Gastric Mucosal NO and $\mathrm{PGE}_{2}$} Contents and Gastric Juice Mucin Concentration in Adult and Aged Rats

From Figure 4, it was clear that CRS produced a significant reduction in the gastric mucosal content of $\mathrm{NO}$ and $\mathrm{PGE}_{2}$ and in the gastric juice mucin level in both adult and aged groups CRS - rats compared to their respective control groups. Moreover, a significant decline was observed in these parameters in aged CRS group compared to adult CRS group.

On the contrary and as shown in Figure 4, pretreatment with CIL produced a significant $(\mathrm{p}<0.05)$ elevation in these parameters in adult and aged CRS + CIL rat groups compared to their respective CRS groups with less significant $(\mathrm{p}<0.05)$ rise in these parameters in aged CRS + CIL group compared to adult CRS + CIL group. 


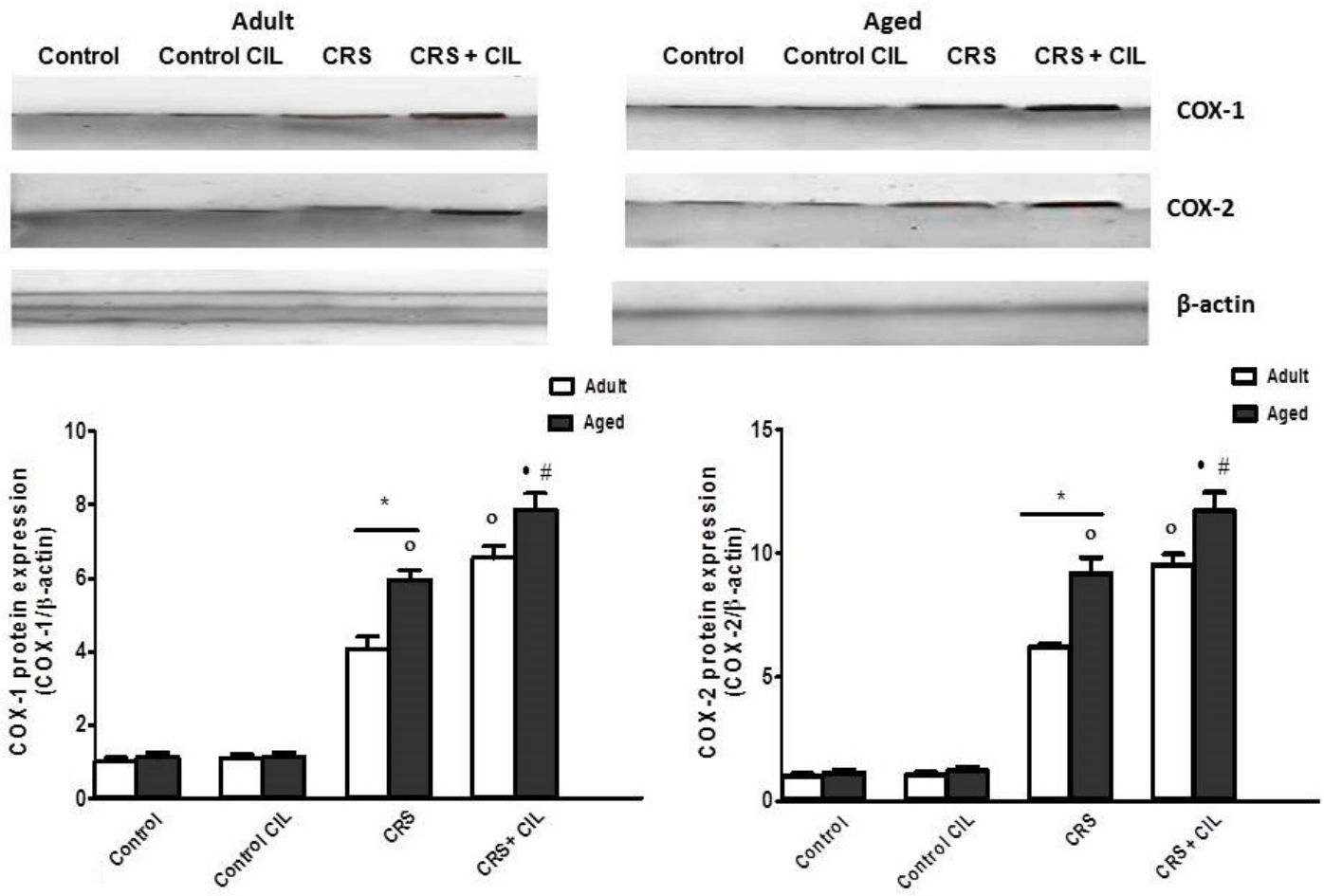

Figure 3. Effect of CIL on gastric mucosal COX-1 \& 2 protein expression in CRS-induced gastric ulcer in adult and aged rats.

Data are mean \pm SEM of 6 rats

*Significantly different from normal control rats at $\mathrm{p}<0.05$.

-Significantly different from adult CRS rats at $p<0.05$.

- Significantly different from aged CRS rats at $p<0.05$.

\#Significantly different from adult CRS $+\mathrm{CIL}$ rats at $\mathrm{p}<0.05$

CRS: Cold restraint stress; CIL: Cilostazol.

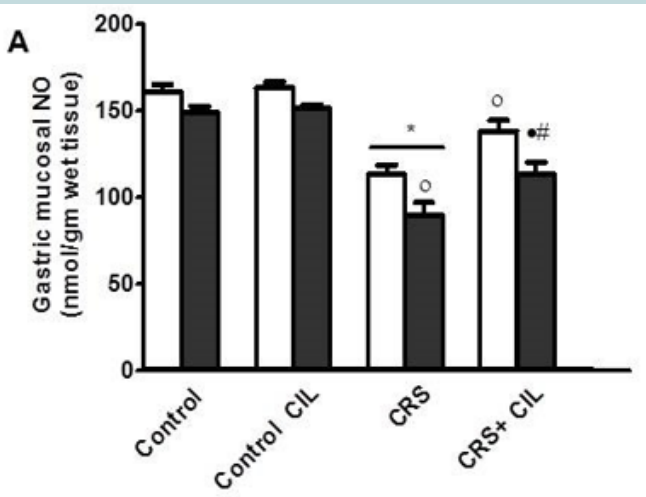

$\square$ Adult
$\square$ Aged
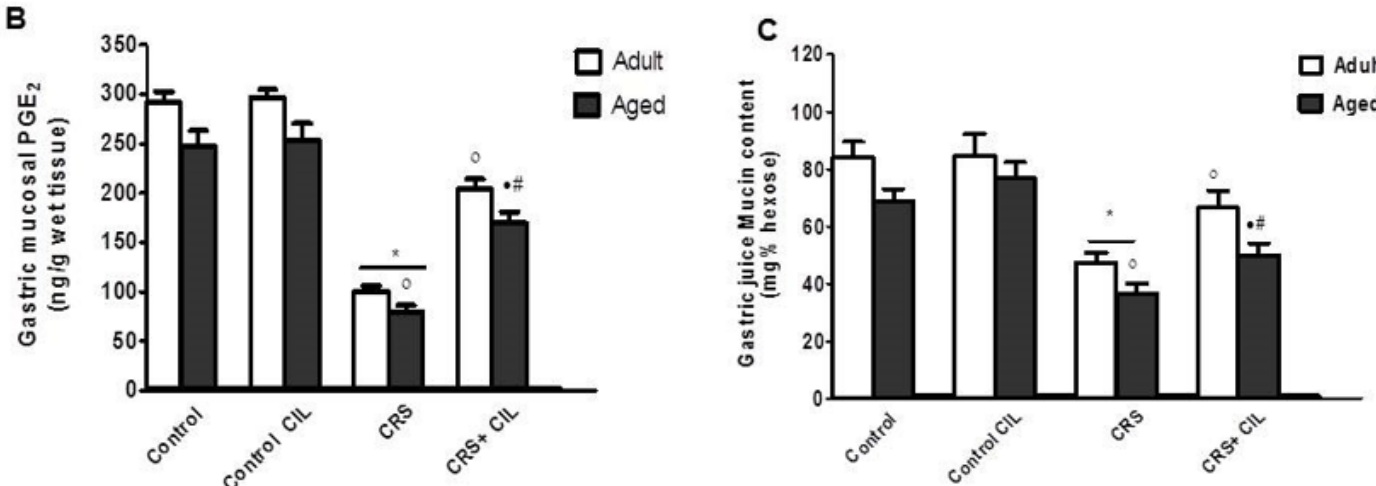

Figure 4. Effect of CIL on gastric mucosal $N O(A)$ and $\mathrm{PGE}_{2}(\mathrm{~B})$ concentrations and gastric juice mucin content $(\mathrm{C})$ in CRS-induced gastric ulcer in adult and aged rats

Data are mean \pm SEM of 6 rats.

"Significantly different from normal control rats at $p<0.05$.

oSignificantly different from adult CRS rats at $p<0.05$

- Significantly different from aged CRS rats at $p<0.05$.

\#Significantly different from adult CRS + CIL rats at $p<0.05$

CRS: Cold restraint stress; CIL: Cilostazol. 


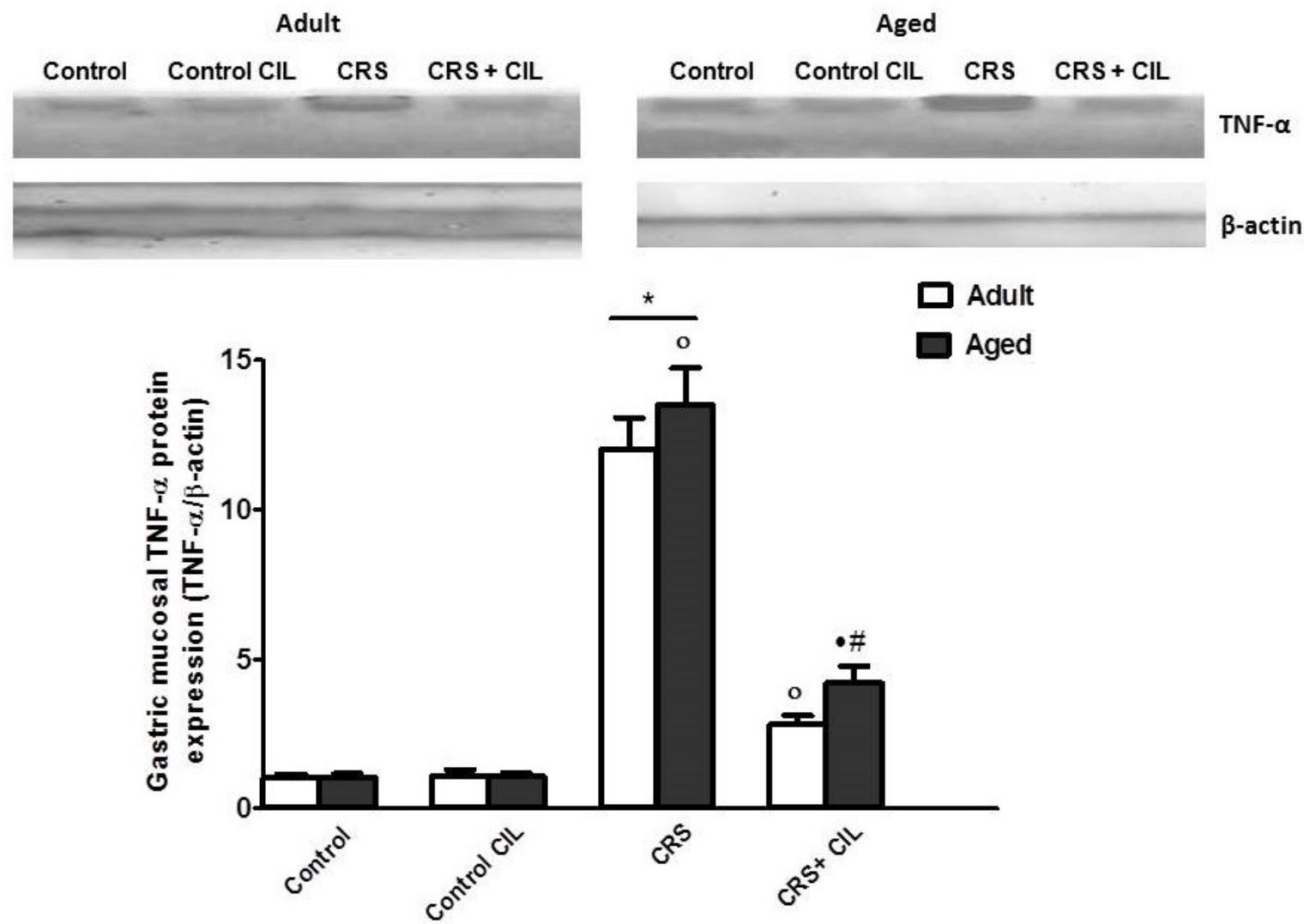

Figure 5. Effect of CIL on gastric mucosal tumor necrosis factor-alpha (TNF- $\alpha$ ) protein expression in CRS-induced gastric ulcer in adult and aged rats

Data are mean \pm SEM of 6 rats.

*Significantly different from normal control rats at $p<0.05$.

oSignificantly different from adult CRS rats at $p<0.05$.

- Significantly different from aged CRS rats at $p<0.05$.

\#Significantly different from adult CRS + CIL rats at $p<0.05$

CRS: Cold restraint stress; CIL: Cilostazol.

\section{Effect of CIL on Gastric Mucosal TNF- $\alpha$ Protein}

\section{Expression in Adult and Aged Rats}

CRS significantly $(\mathrm{p}<0.05)$ augmented the gastric mucosal TNF- $\alpha$ protein expression in adult and aged CRS groups compared to their respective control groups with a more significant rise in aged CRS group compared to adult CRS group (Figure 5). In contrast, CIL pretreatment showed a significant reduction in TNF- $\alpha$ protein expression in CRS + CIL adult and aged groups compared to their respective CRS groups with less significant $(\mathrm{p}<0.05)$ diminution in aged CRS + CIL group compared to adult CRS + CIL group.

\section{Histopathological Results}

Representative photomicrographs display normal thickness of the gastric mucosa of control adult and aged rats (Figure 6A \& B, respectively) and in control adult and aged rats received CIL (Figure 6C \& D, respectively). On the other hand, The histological examinations of both adult and aged CRS groups showed disruption of the gastric gland superficial region with large deep wide mucosal ulcer and inflammation (Figure 6E \& F, respectively). Pretreatment with CIL ameliorated the gastric mucosal damage and inflammatory responses with small shallow mucosal ulcer in both adult and aged CRS + CIL groups (Figure 6G \& $\mathbf{H}$, respectively).

\section{DISCUSSION}

Previous studies have documented several important abnormalities including changes in the structural and functional properties of the gastric mucosa and GI tract in aged people [3,5]. It has been disclosed that aging is an independent risk factor for the development of NSAIDinduced gastric ulceration [21]. This study investigates the protective effect of CIL on experimentally-induced gastric ulceration in adult and aged female rats.

The significant increase in the U.I in aged rats subjected to CRS compared to adult CRS rats suggested that the aged group was more susceptible to peptic ulcer than adult group. The findings of this study are in agreement with the previously reported data of SEO et al. (2012) [4] who indicated that the susceptibility to injury by a variety of damaging agents is increased by aging with impairment of the healing of gastric ulcerations. In addition, many studies reported that the gastric mucosa of aged individuals has noticeable structural and functional abnormalities compared to the gastric mucosa in young people [21,22]. Pre-treatment with CIL ameliorated the U.I and produced a potential 


\section{Adult groups} Control

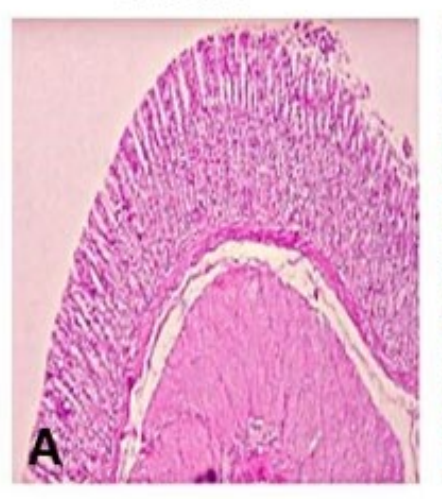

Control CIL

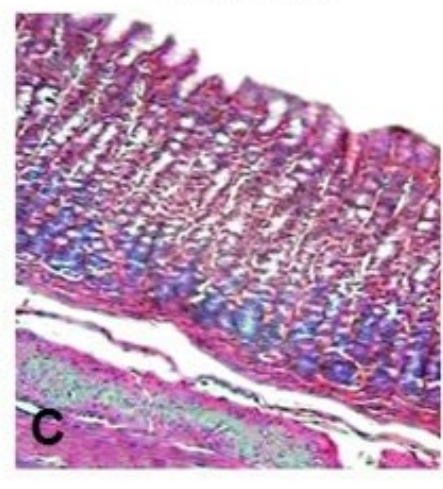

CRS

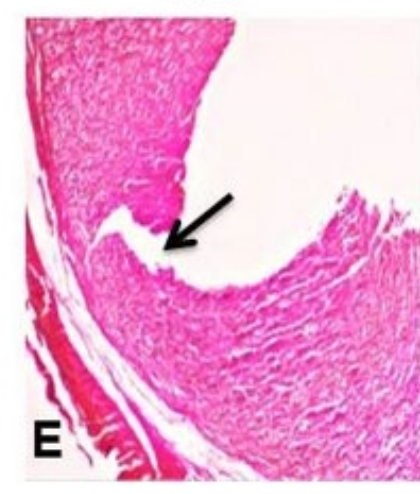

CRS + CIL

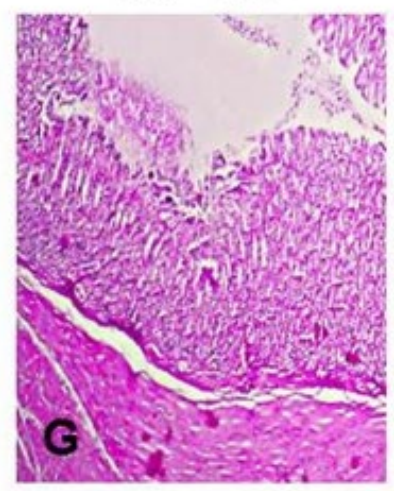

\section{Aged groups}

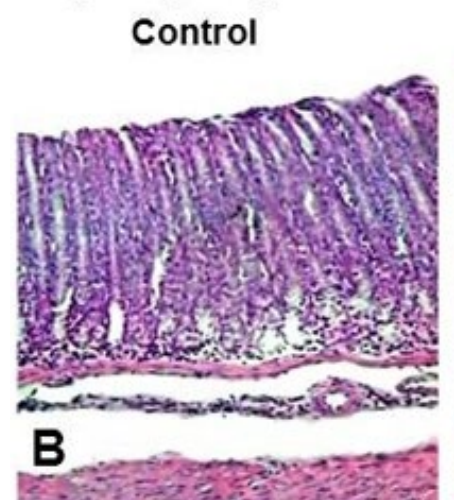

ControlCIL

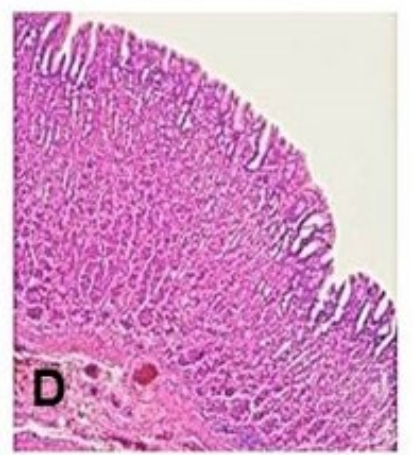

CRS

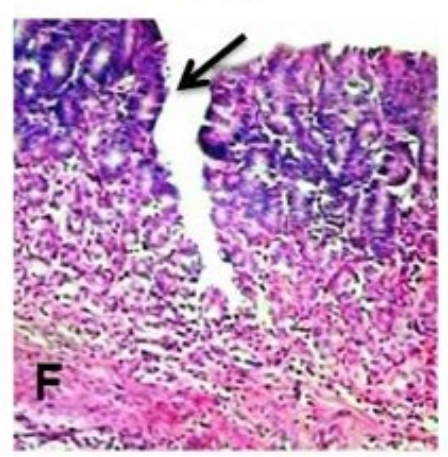

CRS + CIL

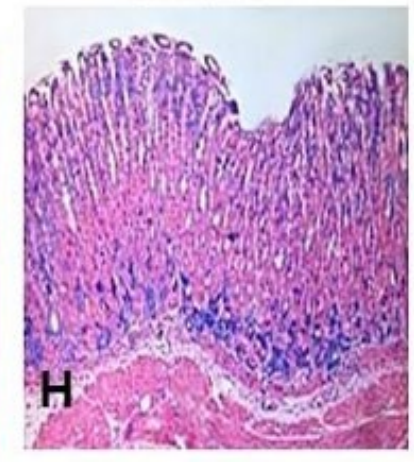

Figure 6. Photomicrographs of the rat gastric mucosa in adult and aged control rats ( $\mathrm{A}$ and $\mathrm{B}$, respectively), adult and aged control CIL rats ( $C$ and $D$, respectively), adult and aged CRS-induced gastric ulcer rats ( $E$ and $F$, respectively), and adult and aged $C R S+C I L$ rats $(G$ and $\mathrm{H}$, respectively).

protective effect in both adult and aged CRS rats compared to CRS model in adult and aged rats, respectively. These findings are in accordance with that of Ohba et al. (2006) [23] who reported that CIL alleviated water-immersion stressinduced gastric inflammation and injury by decreasing the production of proinflammatory cytokines.

The oxidative stress-induced damage, incurred during gastric ulcer, may be related to the accumulation of reactive oxygen species (ROS) with subsequent destruction of epithelial basement membrane [24]. In the current study, the gastric mucosal content of MDA and MPO were increased, while GSH level and GPx activity were decreased in adult and aged CRS rats compared to their control groups with more significant changes in aged CRS rats compared to adult CRS rats. This finding suggests that the stomach in aged rats was already susceptible condition to oxidative stress and coincides with that obtained by Laine et al. (2008) [25] who reported that ROS have been known to be a major risk factor in tissue damage and aging. Pre-treatment with CIL reversed these oxidative changes and suppressed most of the damaging effects induced by CRS. The results obtained in the present study are in harmony with the previously reported data that indicated the anti-oxidant effects of CIL in several experimental models [10,11]. CIL inhibited lipid peroxidation and ameliorated gentamicin - induced oxidative stress possibly by its free radical scavenging property and/or by increasing the activity of the endogenous antioxidants [26]. Additionally, Da Motta and de Brito (2016) [27] reported that CIL decreased lipid peroxidation and improved glutathione level in hypercholesterolemic rats.

The findings of the current study showed that CIL increased gastric mucosal expressions of both COX-1 and COX-2 in adult and aged CRS rats compared to CRS model in adult and aged rats, respectively. Such data indicated that there is cooperation between COX-1 and COX-2 to produce significant contributions to the gastric mucosal defense by CIL. These results are, to some extent, in agreement with the data obtained by Tanaka et al. (2007) [28] who demonstrated that mild stress protects against CRS-induced gastric ulceration by PGs derived from both COX-1 and COX-2 through activation of phospholipase A2. Furthermore, Amagase et al. (2014) [29] revealed that PGs derived from COX-1 play an important role in the gastric mucosal defense during CRS. On the contrary, it has been reported that COX2 plays a crucial role in gastric mucosal defense [30]. Moreover, these results can be explained in the light of the published report indicating that COX-2 is important for the proliferation of epithelial cell, re-epithelization and 
reconstruction of gastric glands during the repair of gastric ulcer [31].

$\mathrm{NO}$ is a potent vasorelaxant mediator involved in the control of gastric blood flow and contributes to the maintenance of gastric mucosal integrity [32]. It was evident that the gastroprotective effect of NO mostly due to the maintenance of blood flow around the ulcer and increase PGE2 production [33]. In addition, PGs are potent vasodilator cytoprotective factors in gastric mucosa that stimulate mucus and biocarbonate secretion, maintain mucosal blood flow and regulate mucosal repair [30]. The findings of this study revealed that the gastric mucosal NO and PGE2 concentrations and gastric juice content of mucin were reduced in both adult and aged CRS rats compared to control groups with more significant decrease in aged CRS group compared to adult CRS group. Both experimental and clinical studies had reported impairment in gastric mucosal defenses by aging as indicated by decrease in bicarbonate, mucus and PGs secretion, reduced NOS activity and reduced blood flow [22,33]. Moreover, Saber and Bayumi (2016) [34] indicated that elderly people have a decreased mucosal PGE2 concentration compared to adults. Our results also indicated that CIL increased gastric mucosal $\mathrm{NO}$ and PGE2 concentrations and gastric juice content of mucin in rats compared to adult and aged CRS groups. NO was indicated to enhance PGE2 biosynthesis via a cGMP-independent mechanism in vivo. It is possible to adopt that NO may regulate the biosynthesis and the release of PGE2 in the gastric mucosa after its injury [33]. Furthermore, NO not only recovers mucosal blood flow but also plays an essential role in encouraging mucus secretion that is one of the constitute of the primary gastric mucosal defense mechanism [35]. Therefore, it could be stated that increasing gastric mucosal level of NO by CIL lead to increased mucosal blood flow and consequently, increased production of gastric mucosal PGE2 and gastric juice mucin content.

The significant increase in gastric mucosal expression of TNF- $\alpha$ in aged CRS group compared to adult CRS group suggests the finding that the stomach in aged rats is exposed to more susceptible condition of inflammations than younger age. Similar results were found in a previous study which indicated that protein expressions of TNF- $\alpha$ and interleukin-1 $\beta$ (IL-1 $\beta$ ) in the gastric mucosa were increased with age [5]. Another study demonstrated that chronic inflammation is considered as an important risk factor underlying aging and age-related diseases [3]. CIL produced significant inhibition in the expression of TNF- $\alpha$ in both age levels, suggesting a possible anti-inflammatory role of CIL which has been demonstrated in previous studies $[23,36]$. CIL decreased stress-induced gastric mucosal inflammation and injury via suppressing the concentrations of MPO, IL-1 $\beta$ and TNF- $\alpha$ on water-immersion stress-induced gastric mucosal lesions [23]. Moreover, Mendes and his co-workers (2009) [36] indicated that CIL inhibits inflammation and fibrosis in sponge-induced peritoneal adhesion in rats via inhibition of TNF- $\alpha$ production.

The histopathological results revealed that pretreatment with CIL produced a structural integrity of the gastric mucosa and supported to the indicative mechanism of action of CIL. Although CIL produced significant protection in both adult and aged rats, it is noteworthy that its protection observed in aged rats was significantly lower than that observed in adult group and this could be attributed to the expected natural abnormalities in aged gastric mucosa. As mentioned previously, these abnormalities may include impairment of gastric mucosal defense (reduction of bicarbonate and prostaglandin production), increasing susceptibility to the injury by damaging agents and reduction of the therapeutic efficacy of anti-ulcer drugs [21,22].

\section{CONCLUSIONS}

Overall, the results of the present work delineate a gastroprotective role for CIL against CRS-induced gastric ulcer in both adult and aged rats. Moreover, the protection afforded by CIL appears to stem from its augmentation of the gastric mucosal defense by its anti-oxidant and antiinflammatory effects.

\section{ACKNOWLEDGEMENT}

The authors express their appreciation to Dr. Esam Omar Kamel, Department of Histology, Faculty of Medicine, Al Azhar University, Assiut, Egypt for his valuable cooperation throughout this work regarding the histopathological part of this work.

Declaration of interest: The authors report no conflicts of interest.

Financial Disclosure: No financial support was received.

\section{REFERENCES}

1. Chang XR, Peng L, Yi SX, Peng Y, Yan J. Association of high expression in rat gastric mucosal heat shock protein 70 induced by moxibustion pretreatment with protection against stress injury. World J. Gastroenterol. 2007;13:4355-9.

2. Dekanski D, Janićijević-Hudomal S, Ristić S, Radonjić NV, Petronijević ND, Piperski V, et al. Attenuation of cold restraint stress-induced gastric lesions by an olive leaf extract. Gen. Physiol. Biophys. 2009;28:135-42.

3. Chung HY, Lee EK, Choi YJ, Kim JM, Kim DH, Zou Y, et al. Molecular inflammation as an underlying mechanism of the aging process and age-related diseases. J. Dent. Res. 2011;90:830-840.

4. Seo PJ, Kim N, Kim JH, Lee BH, Nam RH, Lee HS, et al. Comparison of Indomethacin, Diclofenac and AspirinInduced Gastric Damage according to Age in Rats. Gut and Liver 2012;6:210-217.

5. Choi YJ, Kim N, Lee JY, Nam RH, Suh JH, Lee SM, et al. PMK-S005 Alleviates Age-Related Gastric Acid Secretion, Inflammation, and Oxidative Status in the Rat Stomach. Gut and Liver 2016;15:10:749-756. 
6. Eikelboom JW, Hirsh J, Spencer FA, Baglin TP, Weitz JI. Antiplatelet Drugs: Antithrombotic Therapy and Prevention of Thrombosis, 9th ed: American College of Chest Physicians Evidence-Based Clinical Practice Guidelines. Chest 2012;141:89-119.

7. Sostres C, Lanas A. Gastrointestinal effects of aspirin. Nat. Rev. Gastroenterol. Hepatol. 2011;8:385-394.

8. Patrono C, García Rodríguez LA, Landolfi R, Baigent C. Low-dose aspirin for the prevention of atherothrombosis. N. Engl. J. Med. 2005;353:2373-2383.

9. Rogers KC, Oliphant CS, Finks SW. Clinical efficacy and safety of cilostazol: a critical review of the literature. Drugs 2015;75(4):377-395.

10. Hattori Y, Suzuki K, Tomizawa A, Hirama N, Okayasu T, Hattori S, et al. Cilostazol inhibits cytokine-induced nuclear factor-kappaB activation via AMP-activated protein kinase activation in vascular endothelial cells. Cardiovasc. Res. 2009;81:133-139.

11. Lee WC, Chen HC, Wang CY, Lin PY, Ou TT, Chen CC, et al. Cilostazol ameliorates nephropathy in type 1 diabetic rats involving improvement in oxidative stress and regulation of TGF-Beta and NF-kappaB. Biosci. Biotechnol. Biochem. 2010;74:1355-1361.

12. Odashima M, Otaka M, Ohba R, Jin M, Wada I, Horikawa Y, et al. Attenuation of gastric mucosal inflammation induced by aspirin through inhibition of selective type III phospshodiesterase in rats. Dig. Dis. Sci. 2007;52:1355-1359.

13. Winzler RJ. Determination of serum glycoproteins. In: Glick DP, eds., Methods of Biochemical Analysis. New York: Interscience Publishers Inc., 1955:279-311.

14. Biswas K, Bandyopadhyay U, Chattopadhyay I, Varadaraj A, Ali E, Banerjee RK. A novel antioxidant and antiapoptotic role of omeprazole to block gastric ulcer through scavenging of hydroxyl radical. J. Biol. Chem. 2003;278:10993-1001.

15. Ohkawa H, Ohishi N, Yagi K. Assay of lipid peroxidation in animal tissues by thiobarbituric acid. Anal. Biochem. 1979;95:351-358.

16. Bradley PP, Priebat DA, Christensen RD, Rothstein G. Measurement of cutaneous inflammation: estimation of neutrophil content with an enzyme marker. J. Invest. Dermatol. 1982;78:206-209.

17. Griffith OW. Determination of glutathione and glutathione disulfide using glutathione reductase and 2vinylpyridine. Anal. Biochem. 1980;15:106:207-212.

18. Paglia DE, Valentine WN. Studies on the quantitative and qualitative characterization of erythrocyte glutathione peroxidase. J. Lab. Clin. Med. 1967;70:158169.

19. Green LC, Wagner DA, Glogowski J, Skipper PL, Wishnok JS, Tannenbaum SR. Analysis of nitrate, nitrite, and [' $5 \mathrm{~N}$ ] nitrate in biological fluids. Anal. Biochem., 1982;126:131-138.
20. Bradford MM. A rapid and sensitive method for the quantitation of microgram quantities of protein utilizing the principle of protein-dye binding. Anal. Biochem. 1976;72:248-254.

21. Tarnawski AS, Ahluwalia A, Jones MK. Increased susceptibility of aging gastric mucosa to injury: The mechanisms and clinical implications. World J. Gastroenterol. 2014;20:4467-4482.

22. Kang JM, Kim N, Kim JH, Oh E, Lee BY, Lee BH, et al. Effect of aging on gastric mucosal defense mechanisms: ROS, apoptosis, angiogenesis, and sensory neurons. Am. J. Physiol. Gastrointest. Liver Physiol. 2010;299:11471153.

23. Ohba R, Otaka M, Odashima M, Jin M, Komatsu K, Konishi N, et al. Effect of cilostazol, a selective type-III phosphodiesterase inhibitor, on water-immersion stress-induced gastric mucosal injury in rats. J. Gastroenterol. 2006;41:34-40.

24. Peng YC, Hsu CL, Tung CF, Chou WK, Huang LR, Hung DZ, et al. Chemiluminescence assay of mucosal reactive oxygen species in gastric cancer, ulcer and antral mucosa. Hepatogastroenterol. 2008;55(82-83):770-773.

25. Laine L, Takeuchi K, Tarnawski A. Gastric mucosal defense and cytoprotection: bench to bedside. Gastroenterol. 2008;135:41-60.

26. Ahangarpour A, Heidari H, Mard SA, Hashemitabar M, Khodadadi A. Progesterone and cilostazol protect mice pancreatic islets from oxidative stress induced by hydrogen peroxide. Iran J. Pharm. Res. 2014;13:937-944.

27. Da Motta NA, De Brito FC. Cilostazol exerts antiplatelet and anti-inflammatory effects through AMPK activation and NF-kB inhibition on hypercholesterolemic rats. Fundam. Clin. Pharmacol. 2016;30:327-337.

28. Tanaka A, Hatazawa R, Takahira Y, Izumi N, Filaretova L, Takeuchi K. Preconditioning stress prevents cold restraint stress-induced gastric lesions in rats: roles of COX-1, COX-2, and PLA2. Dig. Dis. Sci. 2007;52:478487.

29. Amagase K, Izumi N, Takahira Y, Wada T, Takeuchi K. Importance of cyclooxygenase-1/prostacyclin in modulating gastric mucosal integrity under stress conditions. J. Gastroenterol. Hepatol. 2014;4:3-10.

30. Wallace JL. Prostaglandins, NSAIDs, and gastric mucosal protection: why doesn't the stomach digest itself? Physiol. Rev. 2008;88:1547-1565.

31. Schmassmann A, Zoidl G, Peskar BM, Waser B, Schmassmann-Suhijar D, Gebbers JO, et al. Role of the different isoforms of cyclooxygenase and nitric oxide synthase during gastric ulcer healing in cyclooxygenase1 and -2 knockout mice. Am. J. Physiol. Gastrointest. Liver Physiol. 2006;290:747-756.

32. Groneberg D, Voussen B, Friebe A. Integrative control of gastrointestinal motility by nitric oxide. Curr. Med. Chem. 2016;23:2715-2735. 
33. Takeuchi K, Kita K, Hayashi S, Aihara E. Regulatory mechanism of duodenal bicarbonate secretion: Roles of endogenous prostaglandins and nitric oxide. Pharmacol. Ther. 2011;130:59-70.

34. Saber A, Bayumi K. Age - Related Gastric Changes. J. Surgery 2016;4:20-26.
35. El-Demerdash E, El-Mesallamy HO, Abu-Zaid NM, Gad MZ. The potential therapeutic effect of nitric oxide modulators in experimentally-induced gastric ulcers. Drug Discov. Ther. 2010;4:276-284.

36. Mendes JB, Campos PP, Rocha MA, Andrade SP. Cilostazol and pentoxifylline decrease angiogenesis, inflammation, and fibrosis in sponge-induced intraperitoneal adhesion in mice. Life Sci. 2009; 10:84(15-16):537-543. 